\title{
Lack of Pharmacokinetic Interaction between Enalapril and Amlodipine with Phenytoin in Rhesus monkeys
}

\section{S.K.Garg, D.K. Badyal \& S Majumdar*}

A cross-over and multiple dose study was carried out to find out possible pharmacokinetic interactions between phenytoin (DPH $35 \mathrm{ma} / \mathrm{kg}$; p.o.) and antihypertensives enalapril (1.6 mg/kg; p.o.) and amlodipine (0.4 mg/kg, p.o.) in rhesus monkeys. Neither the plasma concentrations nor the pharmacokinetic parameters of DPH were altered by coadministration of enalapril or amlodipine, suggesting that enalapril and amlodipine can be safely administered to epileptic patients receiving phenytoin.

Department of Pharmacology \& Experimental Medicine * Postgraduate institute of Medical Education \& Research, Chandigarh- 160012 (India) 